JOURNAL OF EXPERIMENTAL SOCIAL PSYCHOLOGY 6, 26-46 (1970)

\title{
Social Facilitation and Imitation in Group Risk-Taking ${ }^{1}$
}

\author{
Robert B. Zajonc, Robert J. Wolosin, Myrna A. Wolosin, \\ and WaLIace D. LoH \\ The University of Michigan
}

\begin{abstract}
The role of social facilitation and imitation in group risk-taking was explored in two experiments. The first experiment tried to determine if individual risk preferences can be changed by the mere presence of others and if these changes can account for shifts toward risk. Subjects predicted which of two differentially probable stimulus events would occur in a series of 360 trials. Expected value of the choices was held constant by varying the payoff inversely with the frequency of the two events. After 180 trials alone, subjects were put into one of four conditions, Control, Audience, Coaction, and Group. In the two social facilitation conditions, Audience and Coaction, there was an enhancement of dominant responses (i.e., conservative choices). But the Group condition showed a change in the risky direction, indicating that social facilitation processes cannot account for risky shifts. Experiment II replicated the social facilitation effects found in Experiment $\mathrm{I}$, but failed to find pronounced tendencies of individuals to make their choices consistent with those of others. These weak imitation effects were found for a situation in which only knowledge of others' choices was accessible to the subject and in a situation in which both this knowledge was accessible and others were actually present. The implications of these findings for theories of risky shift were discussed.
\end{abstract}

The comparison of individual and group decision-making under uncertainty and risk has received much attention in recent years. Most studies indicate that groups tend to take greater risks than individuals, and a rather large number of hypotheses has been offered to account for this phenomenon (Bateson, 1966; Brown, 1965; Burns, 1967; Flanders \& Thistlethwaite, 1967; Kogan \& Wallach, 1967; Marquis, 1968; Pruitt \& Teger, 1968; Stoner, 1968; Teger \& Pruitt, 1967; Wallach, Kogan \& Bem, 1962; Zajonc, Wolosin, Wolosin, \& Sherman, 1968, 1969). But none of these hypotheses has enjoyed unequivocal support. The controversy

${ }^{1}$ These experiments were made possible by Grant GS-629 from the National Science Foundation. We wish to thank Philip Brickman, John Forward, Ed Lichstein, and Jean-Pierre Poitou for their help in the various phases of the experiments. 
which developed in connection with the risky shift phenomenon has been exacerbated by two assumptions, both probably false, that seem to have been implicitly made by some parties to the controversy. They are: (a) a shift toward risk is an inevitable consequence of making decisions in groups; $(b)$ there exists a unique necessary antecedent condition which when discovered will explain this phenomenon completely. And the controversy has centered over the nature of this unique antecedent.

That the first assumption is false is indicated by such recent results as those of Burns (1967), Marquis (1968), Marquis and Reitz (1968), Nordhøy (1962), Rabow, Fowler, Bradford, Hofeller, and Shibuya (1966), Stoner (1968), and Zajonc et al. (1968). In these experiments a shift toward caution was evidenced. That there does not exist a unique necessary antecedent condition for the occurrence of a shift toward risk, but, on the contrary, that there are many diverse sufficient conditions, is suggested by the fact that all of the seemingly conlicting hypotheses have received some support. Thus, for instance, the theory which holds that groups take greater risks than individuals because the responsibility for outcomes is diffused among the group members received support from experiments by Wallach et al. (1964) and by Rettig, Johnson, and Turoff (1967), although the latter authors found that a shift toward riskier actions occurs only if the responsibility can be shared with others who are known by the subject and who form a cohesive group with him. The hypothesis that the group situation allows the individual to become more familiar with the problem has been supported by Bateson (1966) and by Flanders and Thistlethwaite (1967). And the various theories involving cultural values (Brown, 1965) have also received some support (Hinds, 1962; Stoner, 1968).

If it is assumed that a shift toward risk occurs not as a result of one unique necessary antecedent condition, but that there exist several preconditions which may lead to such a shift, and that there are others which may lead to a shift toward caution, then the controversy which has grown over the explanation of the phenomenon might be supplanted by a research effort directed at the discovery and enumeration of the various sufficient conditions of risky and conservative shifts. This paper is concerned with one set of such sufficient conditions.

Nearly all theories which deal with the shift toward risk assume that group decisions are riskier (or more conservative) because the group situation changes the risk preferences, or at the minimum the choice behavior, of the individual members. However, it has never been unequivocally shown that individual risk preferences (or choice behavior) change when a person becomes a group member, or that either of these can be changed in any way by social means. A paper by the late Andrew Blank 
(1968) suggests that individuals do make riskier bets in the presence of others engaged at the same task than they make in the presence of the experimenter. But in that study, the risk of the bet was confounded by its expected value, such that the expected value of the riskiest bet was six times higher than the expected value of the most conservative bet. It is, therefore, impossible to determine whether changes toward lower probability bets indicated increased risk preference or increased rationality. Moreover, factors such as competition, conformity, and social comparison might have interacted with the confounding mentioned above to produce the difference between individual and group betting.

To see how changes in individual risk-taking tendencies might be brought about, let us assume that the sheer physical presence of another person increases the drive level of the actor. Support for this assumption derives from a recent review of the social facilitation literature (Zajonc, 1965 ) in which it is assumed that the presence of others acts as a source of general arousal (D) and that it enhances the emission of dominant responses. Most of the evidence supporting the social facilitation hypothesis comes from studies in which performance or learning is observed under social conditions and under isolation (Cottrell, Rittle, \& Wack, 1967; Zajonc \& Sales, 1966). But there has also been some evidence for social facilitation effects on choice and preference. Strain (1967) found that the preference of children for oddity is enhanced in the presence of others, and Goldman (1967) has shown that preference for a specific color can also be enhanced under these conditions. Thus, the social facilitation paradigm provides a means for exploring changes in individual risktaking tendencies. Since social facilitation is assumed to operate whenever people act in the presence of others, it also applies to differences in individual and group risk-taking. In regard to the latter, a social facilitation hypothesis would call for groups to shift in a risky direction when the group members are risky when acting independently, but it would predict conservative shifts when members are initially conservative. It is interesting in this respect that since the bulk of the literature indicates a shift toward risk in groups, we would expect the preference of individuals tested alone to be on the risky side of the continuum. This seems to be the trend (see Zajonc et al., 1968, p. 93). Moreover, in cases where the group members are initially cautious, a shift toward conservatism is observed. These findings, however, are not a function of individual difference variables but of situational factors. They come from studies in which items for decisions are classified according to whether they generate initial risk or caution in the individuals. Thus, for instance, Stoner (1968) compared group decisions on life dilemmas which invite risk with those which invite caution when they are considered by the individuals working independently. Shifts toward risk were found for the former 
and toward conservatism for the latter items when these individuals were formed into groups.

This paper reports two expcriments in which wo attcmpt to ascertain whether or not individual decisions are subject to some basic social infuences, such as the effects of the mere presence of an audience or of coactors. In addition, an effort is made to determine if a tendency to conform to the responses of others or to imitate them is a part of the group process presumed to influence the individuals' choices. Finally, it is the purpose of this study to ascertain whether such effects, if they do occur, can account for differences between individual and group risk-taking.

As in our previous studies (Zajonc et al., 1968, 1969), a two-choice situation was used in which the subjects were presented with two events, each having a determined probability of occurrence on a series of 360 trials. The subjects' task was to predict which of two lights would go on on each trial. They were rewarded proportionately more for predicting correctly the infrequent event than for predicting correctly the infrequent event, such that the expected values of the two predictions were equal. This situation differs from the one used in much of the previous research on risk-taking in groups. The advantages of this method and the ways in which it differs from the life dilemma task were discussed in a previous paper (Zajonc et al., 1968, pp. 95-96). It may be argued that winning a few pennies on each of 360 trials may be subjectively so trivial to the person that his "true" risk behavior has no opportunity of revealing itself. On the other hand, theories of group risk-taking do claim a generality extending beyond the life dilemma task. Moreover, there is no reason to believe that the life dilemma task, in which the subject advises a hypothetical person about some hypothetical choices in a hypothetical situation, is subjectively less trivial than one in which he can accumulate a bit of real money. If results obtained in the present study are not consistent with previous research using the life dilcmma task, the generality of empirical work and of theorizing in the area of group risk-taking may be questioned.

\section{EXPERMENT I}

The main purpose of this experiment is to determine whether the presence of coactors and of passive spectators constitutes a sufficient condition for the occurrence of changes in individual choice behavior under uncertainty and risk. It is also our attempt to determine whether such changes in individual decisions, if they do occur under these minimal social conditions, account for the differences that arise when the same decisions are reached in groups. 


\section{METHOD}

\section{Subjects}

One hundred and thirty-two male undergraduate subjects were recruited from the paid psychology subject pool. A minimum of $\$ 1.25$ per hour was guaranteed. When scheduled, subjects were told neither the purpose of the experiment nor that they conld earn a greater sum of money during its course.

\section{Apparatus}

Two 1/25-watt lights, mounted on a small panel, served to signal stimulus events. One light was marked either 1 cent or ${ }^{3 / 4}$ cents. 'I'he other was marked either $1 / 1 / 2$ cents or 3 cents. For half of the subjects the left stimulus light was associated with the higher payoff, and for the remainder, with the lower payoff. A punched-tape program controlled the timing and sequence of stimulus events. Subjects signaled their responses by means of switches and were given poker chips to tally their winnings. Stimulus events and responses were recorded on an Esterline-Angus Event Recorder.

\section{Design and Procedure}

All subjects were given 360 trials in 12 blocks of 30 trials each. There were two stimulus conditions to which subjects were randomly assigned:

.6-.4 Condition. The two stimuli were programmed with .6 and .4 probabilities of occurrence. The payoffs for this condition were 1 cent and $1 / 2$ cents, respectively.

.8-.2 Condition. ${ }^{2}$ The stimuli were programmed with probabilities of occurrence equal to .8 and 2 . In this condition the payoffs were ${ }_{4}^{\prime \prime}$ cents and 3 cents. The sequence of stimulus events was random, with the constraint that one or the other of the above probabilities would be observed in each block of 30 trials.

Upon arriving at the laboratory, subjects were met by the experimenter and taken to their individual cubicles where they all worked alone for the first 180 trials. After a short while, the following taped instructions were given over an intercom system. (The instructions reproduced here refer to the .6-.4 condition. For the .8-.2 condition, the words “3/4 $(3)$ cents" were substituted in the appropriate slots.)

In front of you, you see two lights. Every 7 seconds one or the other will go on. You also see two response plates. During each 7-second interval you are to press one plate or the other to anticipate which light will come on. If you press the left plate and the left light comes on, you win 1 (one and a half) cents. If you press the right plate and the right light comes on, you win $1 \frac{11}{2}$ (one) cents. If you press one plate and the opposite light comes on you win nothing. Also, if you fail to press within the 7-second interval, you win nothing.

You can keep track of your winnings by the chips which you see in front of you. If you win 1 cent by anticipating the left (right) light, place a white chip into your bank. If you win $1 \frac{1}{2}$ cents by anticipating the right (left) light, place a blue chip into your bank. Your winnings will also be kept automatically. We guarantee you a minimum of $\$ 1.25$ per hour and you get to keep whatever you win over that amount; that is, you will get $\$ 1.25$ per hour, or your winnings, whichever is more.

Stimuli were presented every 7 seconds and there was a 1-minute rest period after each trial block. Blue poker chips were always associated with the high payoff light,

${ }^{2}$ The results for condition .8-.2 were reported at the 1968 meetings of the Midwestern Psychological Association in Chicago. 
and white poker chips, with the low payoff light. Subjects were randomly assigned to one of four treatments within each of the two stimulus conditions.

IA treatment ( 24 subjects). At the end of the sixth trial block subjects in the Individual-Audience (IA) treatment were told that there was a student interested in viewing the experiment. If the subject didn't mind, this student would join him for the remainder of the session. Upon obtaining the subject's permission, the audience took a place in the cubicle behind him. The subjects and the spectators were warned not to speak with each other. The subject then continued as previously for 180 trials.

IC treatment (24 subjects). At the end of the sixth trial block sabjects in the Individual-Coaction (IC) treatment were told that due to scheduling difficulties, they could no longer continuc to work in their individual cubicles. They werc taken in groups of three to a larger room where identical apparatus had been set up in booths. Each subject was seated at a booth where he could not see the others, and continued responding for another set of 180 trials. The subjects were warned not to speak with each other.

II treatment ( 36 subjects). At the end of the sixth trial block subjects in the Individual-Individual (II) treatment were told that the first part of the experiment was over. They were then taken out of their cubicles by the experimenter and allowed to relax for about 3 or 4 minutes. They were not permitted, however, to speak to one another. After the rest period, they were ushered back to their cubicles and continued as previously for six blocks of 30 trials.

$I G$ treatment ( 48 subjects). ${ }^{3}$ At the end of the sixth trial block swbjects in the Individual-Group (IG) treatment were told that the first part of the experiment was over. The experimenter then met all three subjects and led them to a room equipped with apparatus identical to that which they had used alone. Taped instructions were again issued over the inlercon:

Again you see the lights and the plates. Now you are working as a team. This time one or the other light will go on every 20 seconds. During this interval the threc of you will work together to come to a manimous decision as to which plate to press. After you decide, one of you will press the plate. We will tell you who will begin pressing, and we will also tell you when to switch. Once again, if you press the left plate and the left light comes on, each of you wins 1 (one and a half) cent. If you press the right plate and the right light comes on, each of you wins $l^{1 / 2}$ (one) cents. If you press one plate and the other comes on, or if you fail to press within the 20-second interval, each of you wins nothing. Either you all win or all lose on every trial. Again, you can keep track of your winnings by placing the appropriate chips in your bank.

Six blocks of 30 trials, each separated by 3-minute intervals between the blocks, were given. As announced to the subjects, there was a 20 -second intertrial interval. Each subject pressed the plates during two of the six intervals.

After the experimental session the subjects were asked to make three estimates: (a) "In your estimation, what percentage of the time were you pressing the right hand switch?" (b) "In your estimation, what percentage of the time would the average student at UM press the right hand switch?" (c) "Finally, in your estimation, what percentage of the time would a professional gambler press the right hand switch?" They were provided with linear scales ranging from $0 \%$ to $100 \%$ in $10 \%$ steps.

${ }^{3}$ The two control groups (II and IG) were also employed in another experiment, run simultaneously with Experiment I (Zajonc et al., 1969). 
An explanation of the experiment was then given, and the subjects were paid their wages and their winnings. They were asked not to divulge the details of the experiment to their friends or acquaintances.

\section{RESULTS}

We shall first examine the effects of audience and coaction. As in the previous study (Zajonc et al., 1968), the dependent measure consisted of the percentage of risky choices made on each block of 30 trials. Figure 1 shows, for all conditions and treatments, the percentage of risky choices over the 12 trial blocks. Of immediate interest are the IA (IndividualAudience) and the IC (Individual-Coaction) treatments as they compare with control subjects who continued to perform in isolation throughout the entire experiment (II). These results were evaluated by an analysis of variance in which the between-subject factors were the two stimulus conditions (.6-.4 and .8-.2) and three experimental treatments (II, IA, and IC), while the within-subject factor was trial blocks, with the first and second sets of 180 trials nested within trial blocks.

Two effects are apparent. First, there is a greater number of risky

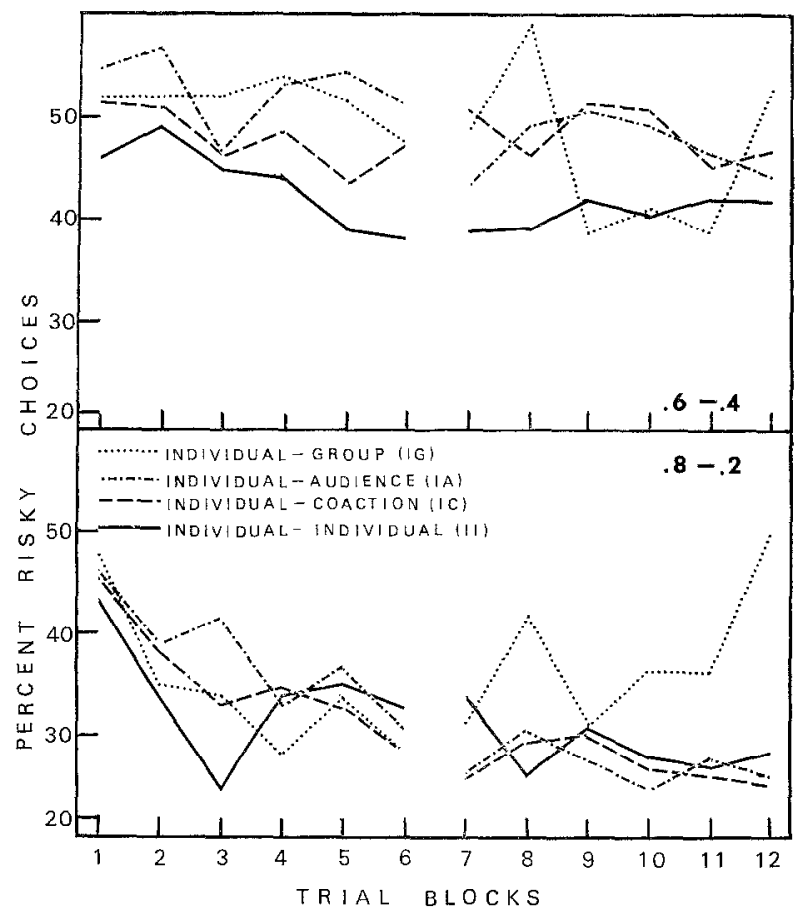

FIG. 1. Percent of risky choices under conditions of social facilitation and in groups. 
choices in the .6-.4 condition than in the $.8-.2$ condition. For example, in the II treatment the asymptotic percentage of risky choices is 40.9 in the .6-.4 condition, but only 28.9 in the $.8-.2$ condition. This difference across all three treatments is significant at the .001 level $(F=21.90,1$ and 78 $d f)$. The second result of interest concerns the differences in the percentages of risky choices between the first and second halves of the experimental session. This difference across all treatments and conditions is also significant ( $F=42.32,1$ and $858 d f, p<.001$ ). In addition, however, we note that in comparison with the $.6-.4$ condition, there is a substantial reduction in risky choices from the first to the second set of 180 trials in the $.8-.2$ condition. These results are seen more clearly in Table 1 , which presents average data for the first and second 180 trials. The relevant interaction term was significant at the .001 level $(F=12.18$, 1 and $858 d f$ ). It should be noted, however, that this interaction is due in part to the relatively high proportion of risky choices in the $.8-.2$ condition in the initial trials. Subjects in this condition, like those in the .6.4 condition, show little preference for one or the other alternative at the beginning of the session: their choices vary between $45 \%$ and $55 \%$. But while the $.6-4$ groups remain near that level throughout the experiment, there is a significant drop in risk in the $.8-.2$ condition.

The inspection of cell means in Table 1 reveals that on the second 180 trials there was a reduction in risky choices in the IA and IC treatments in the $.8-.2$ condition. It is true that there is a drop in risk from the first to the second 180 trials for the $.8-.2$ control subjects as well. However, this drop does not reach an acceptable level of significance, and it probably reflects merely the higher initial risk levels of this group. But the

'TABLE' 1

Avmage Percentage of Risky Choices of Subjects Working in Isolatiox, Coaction, or in the Presence of a Spectator

\begin{tabular}{lcc}
$\begin{array}{c}\text { Condition and } \\
\text { treatment }\end{array}$ & First 180 trials & Second 180 trials \\
\hline $6-.4$ Condition: & $42.8_{\mathrm{de}}{ }^{2}$ & $40.9_{\mathrm{cde}}$ \\
II treatment & $52.8_{\mathrm{f}}$ & $47.1_{\mathrm{ef}}$ \\
IA treatment & $48.0_{\mathrm{ef}}$ & $48.5_{\mathrm{ef}}$ \\
IC treatment & $34.0_{\mathrm{abe}}$ & $28.9_{\mathrm{ab}}$ \\
$.8-.2$ Condition: & $38.1_{\mathrm{cd}}$ & $27.0_{\mathrm{a}}$ \\
II treatment & $35.7_{\mathrm{bcd}}$ & $27.5_{\mathrm{a}}$ \\
IA treatment & \\
IC treatment &
\end{tabular}

* Means having different subseripts are different at the .05 level by a Newman-Keuls comparison. 
conclusions about the effectiveness of the audience and coaction effects must be restrained because of the lack of significant differences among the II, IA, and IC groups during the second 180 trials. Such differences would provide especially strong evidence for a social facilitation effect.

In none of the treatments in the .6-.4 condition was there a significant change from the first to the second 180 trials. It should be noted that when the frequencies of the two alternatives do not substantially differ from each other, as in the $.6-.4$ condition, preferences for these alternatives also do not differ from each other. The largest departure from indifference is $9.1 \%$. Hence, the dominant response for the $.6-.4$ subjects, while stronger than its subordinate counterpart, is quite weak in comparison with the dominant response in the .8-.2 groups. Here the cautious alternatives are selected on 2 out of 3 trials. We would, therefore, expect, according to the social facilitation theory, that the presence of coactors or spectators would act to enhance the preferred choices, and, within the limits of a ceiling, to enhance it more when the preference is pronounced.

Do changes in risky choices obtained in the presence of coactors and of spectators explain shifts toward risk and caution found when individuals make decisions in face-to-face groups? Figure 1 includes the data for the IG treatment and Table 2 summarizes them over the two halves of the experimental session. It is clear from Figure 1 and from Table 2 that whatever process takes place in the group situation, it probably is not merely one of social facilitation. The IG treatment differs from the others in several respects. First, there is considerable variability over trial blocks during the second 180 trials. Second, while there is a drop in risky choices in the .6-.4 condition (a result replicating the previous finding of Zajonc et al., 1968), the percentage of risky choices increases in

\section{TABLE 2}

Average Percentage of Risky Choices of Subjects Working in IsOlation and in Grotps

\begin{tabular}{lcc}
\hline $\begin{array}{c}\text { Condition and } \\
\text { treatment }\end{array}$ & First 180 trials & Second 180 trials \\
\hline 6-.4 Condition & & $40.9_{\mathrm{de}}$ \\
II treatment & $42.8_{\mathrm{ef}}^{*}$ & $16.4_{\mathrm{f}}$ \\
IG treatment & $51.7_{\mathrm{g}}$ & $28.9_{\mathrm{a}}$ \\
$.8-.2$ Condition & $34.0_{\mathrm{b}}$ & $37.7_{\mathrm{ed}}$ \\
II treatment & $34.8_{\mathrm{bc}}$ & IG treatment \\
\hline
\end{tabular}

* Means having different subscripts are different at the .05 level by a Newman-Keuls comparison. 
the $.8-.2$ condition. An analysis of variance on data in Table 2 was performed, again using the two halves of the experimental session as a factor nested within trial blocks. This time, however, the degrees of freedom were reduced in order to equate the reliability of observations in the II and the IG treatments. The data points in this analysis consisted of groups of three subjects in both treatments. In the II treatment random "groups" of three subjects were composed, and the average responses of the members of these "groups" were treated in the same manner as the group responses of the IG groups. This analysis revealed a significant difference between the IG and II treatments $(F=7.07,1$ and $24 d f, p<$ .05 ), a significant difference between conditions ( $F=27.71,1$ and $24 d f$, $p<.001$ ), a significant effect over the 12 trial blocks ( $F=2.34,11$ and $264 d f, p<.01$ ), and, finally, a just significant interaction among treatments, conditions, and sessions $(F=3.85,1$ and $264 d f, p<.05)$. The overall picture then is that in the $.6-.4$ condition the IG subjects behave differently from the II subjects, while the subjects in the social facilitation treatments (IA and IC) behave like the II controls. In the $.8-.2$ condition the IG subjects also behave differently from the II subjects, as do the IA and IC subjects. The subjects working in groups increase their risky choices, and those working in coaction or in the presence of an audience decrease them. It is clear, therefore, that the sort of change in individual risk-taking that is produced by social facilitation cannot be responsible for group effects. In fact, some credence is lent to the view that the process by which groups come to decisions must be strong enough to overcome the effects of social facilitation. What can this process be? While we can only speculate at this point, it might well be that changes in individual risk preferences from alone to group are only part of the story. The other part has to do with how these risk preferences are reconciled by the group in order to arrive at a group decision. By adopting various decision schemes, a group can combine the preferences of its members to becomc more or less risky than the mean riskiness of its members taken individually. In this study, the group decision scheme in the IG treatment, as well as the risk preferences of the group members, was left to vary freely.

There is another way in which the social facilitation groups differ from the IG treatments. In the IG treatment the individual members often announce their preferences. This knowledge is inaccessible to the IA or the IC subjects. Hence, tendency to conform or to imitate may also be part of a process occurring in the group which is responsible for shifts. Our second experiment was carried out in an attempt to determine if the responses of other subjects play a significant part in the individual's choice behavior. 


\section{EXPERIMENT II}

As just mentioned, the preferences of the group members are accessible to all in the IG condition. In the studies of coaction performed in the early thirties this was also true. However, in Experiment I the IC subjects worked in ignorance of each other's choices. Since most of the work on shift to risk involves considerations about the forces that emerge in groups in which all members have knowledge of each other's preferences, the role of pressures toward conformity or tendency toward imitation becomes a significant factor in group decision-making. We shall attempt to establish whether conformity pressures which agree with the subject's preferences and pressures which disagree with his preferences have an effect on his decision behavior. It is of interest in this study, also, to discover if conformity effects interact with social facilitation effects.

Since we are interested in effects on subjects' preferences, we have chosen only the $.8-.2$ condition since subjects in this condition showed the most marked departure from indifference. Also, while unlikely, it is possible that the results of the previous experiment were in part due to the fact that the manipulation of social facilitation occurred after the subject had been in the laboratory for some time. It is possible that the results were due to a disruption of his pattern of responding by the introduction of a spectator or of coactors. Because the previous results were fairly weak, it is especially important to determine if the social facilitation effect can be demonstrated less equivocally. In order to determine if the timing of the social facilitation manipulation is indeed a significant factor, we first conducted a preliminary study in which subjects worked the entire 360 trials in isolation or in the presence of an audience. The second part of the study deals with the interaction between coaction effects and knowledge of others' responses.

\section{METHOD}

\section{Subjects}

Thirty-eight female subjects from the subject pool maintained by the Psychology Department at the University of Michigan took part in the experiment. In contrast with the previous study, these subjects were not guaranteed minimum pay for their participation. By taking part in it they fulfilled a requirement in the introductory psychology course. However, they were paid their winnings.

\section{Apparatus}

The same apparatus as in the previous experiment was used for generating stimuli, one of which occurred $80 \%$ of the time and the other $20 \%$ of the time. The same punchcd-tape program was employed. However, the subjects indicated their choices not by pressing switches, but by announcing them into a microphone and writing them down on a score sheet especially provided for that purpose. The score sheet 
contained 12 pairs of columns, each pair labeled "Left" and "Right." The sheet was divided into 30 rows, each row representing a single trial. The subjects indicated their choices by checking either "Left" or "Right" in the appropriate row and column.

\section{Design and Procedure}

All subjects were given 360 trials in 12 blocks of 30 trials. There was a 3 -minute break after the 180th trial, and the subjects were allowed to leave their cubicles but not to talk with each other. For 20 subjects ( 10 in each group) the frequent stimulus occurred on the right side of their panels, and for 18 on the left side. Nineteen subjects worked in isolation for the entire 360 trials, and 19 worked in the presence of a spectator who was introduced in the same manner as in the previous experiment, except that the spectator was introduced not after 180 trials but immediately after the subjects became familiar with their task. The spectator was also a female student.

The payoffs associated with the frequent and infrequent stimuli were $1 / 2$ cents and 2 cents, respectively. Below are the instructions which were issued to the subjects after they were placed in their individual cubicles:

In front of you, you see two lights. Every 7 seconds, one or the other light will go on. During each 7-second interval, you are to guess which light will come on. You are to make your guess out loud by saying "right" or "left" into the microphone, and then placing a check mark on the answer sheet in the appropriate "right" or "left" box.

If you guess "left" and the left light comes on, you win t/2 cent. If you guess "right" and the right light comes on, you win 2 cents. If you guess one light and the opposite light comes on, you win nothing. Also, if you fail to make a guess within the 7 -second interval, you win nothing. You must make a guess hefore the light comes on.

You can keep track of your winnings by means of the poker chips which are on the table. If you win 2 cents by anticipating the right light, place a blue chip into your box. If you win $\frac{1 / 2}{12}$ cent by anticipating the left light, place a white chip into your box.

In all other respects the subjects were treated as in the first experiment. At the cluse of the session an oral and written description of the study and its purposes was provided. The subjects were asked not to divulge their participation to others.

\section{RESULTS}

\section{The Effects of Audience on Choice Behavior}

The results were treated as in the previous experiment in terms of percentage of risky choices on each of the 12 trial blocks. Figure 2 shows the percentage of risky choices for subjects who worked alone and for: subjects who worked in the presence of an audience. Neither of the groups of subjects shown in Figure 2 had any information or cues about the choices of others. It is clear from Figure 2 that the social facilitation effect is rather pronounced. The changes in the procedure did not affect the results, which are quite similar to those found in the first experiment. Again, on the initial trials the subjects do not manifest preferences for 


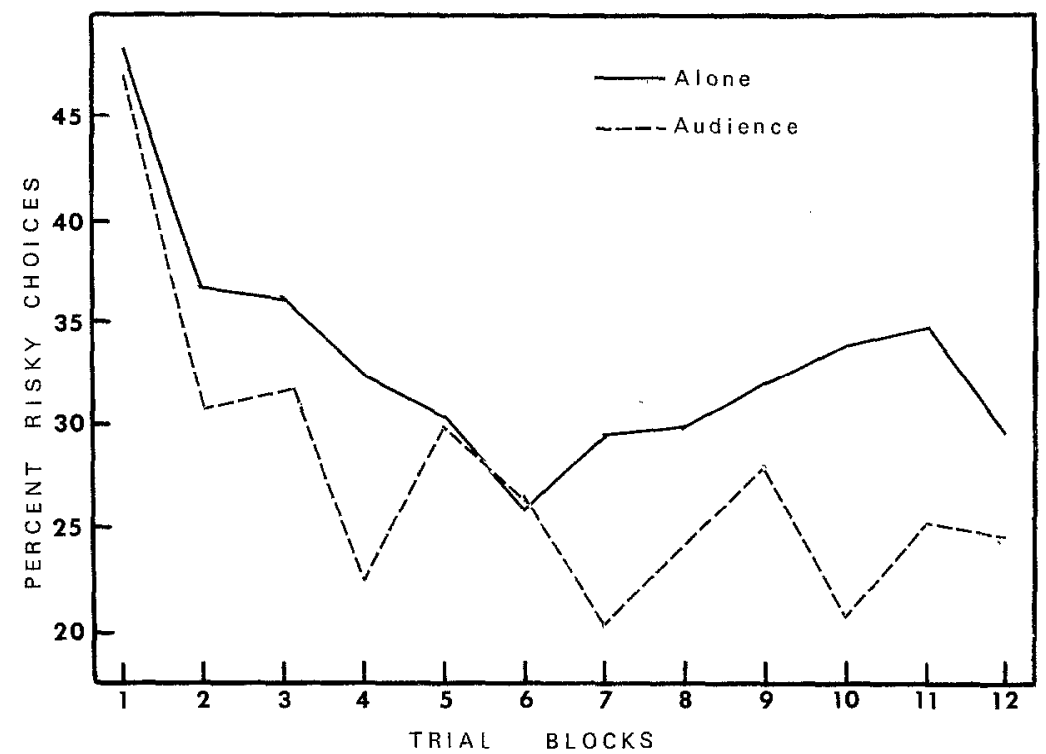

Frg. 2. Percent of risky choices for subjects working alone and in the presence of an audience.

one or the other alternative, the percentage of risky choices on the first trial block being nearly $50 \%$ for both treatments. However, the asymptotic level of risk is considerably lower than that. An analysis of variance was performed in which the treatments (Alone vs. Audience) and trial blocks were orthogonal factors, and in which, for the purpose of comparison with previous studies, the first 180 trials were compared with the second 180 trials as a nested factor. This analysis revealed a significant change over trial blocks ( $F-13.42,11$ and $396 d f, p<.001$ ), a significant difference between the first and second halves of the experiment ( $F=32.15,1$ and $396 d f, p<.001$ ), and a significant interaction between the treatments and halves $(F=4.97,1$ and $396 d f, p<.05)$. During the second 180 trials the subjects working alone averaged $31.6 \%$ risky choices while those working in the presence of an audience averaged only $23.8 \%$. A Newman-Keuls test shows these means to be different at the .05 level. These differences are clearer than in the previous experiment. It may now be concluded with greater certainty that if there exists a dominant preference, this preference will be enhanced in the mere presence of others, as predicted by the drive theory of social facilitation.

\section{The Effects of Cues and Coaction}

The second concern of the present experiment is with the presence of others coupled with the subject's knowledge of their responses. It might 
be expected that if mere presence enhances dominant responses, and if there exists a tendency to imitate others or to conform to what seems to be the group norm, then the combined effects of coaction and of the knowledge of others' choices should have a greater impact on the subject's behavior than the effects of presence alone. It would also be expected that when the behavior of others shows preference opposite to that of the subject, the effect of presence and of others' responses would work against each other. Which of these effects is more resilient depends on the specific manner in which these two variables are manipulated.

\section{METHOD}

\section{Subjects}

Sixty-six subjects recruited in the same manner as in the preliminary part of this study were employed. Ten additional subjects, also females, were recruited from the paid subject pool. They were paid $\$ 1.35$ for participation.

\section{Apparatus}

The apparatus and the punched-tape program were the same as in the preliminary study.

\section{Procedure}

Thirty-eight subjects were randomly assigned to the Coaction condition and 38 to the Alone condition. In addition to the instructions issued to the subjects in the preliminary study the present subjects also were given the following:

Please put on the earphone-microphone system. It is hooked up to a central tape-recording system. Since different microphone channels all come into this same system, it is necessary that responses be made in order. You are on channel two, which means you should be the second person to respond. Therefore, please make your guess immediately after the first person has responded.

In the Coaction condition the subjects worked in the presence of a paid participant who was trained to make the appropriate number of risky and conservative choices. Of the 38 Coaction subjects 19 heard the paid participant make congruent choices and 19 heard her making incongruent choices. Thus, in the Congruent treatment the paid participant chose the frequent alternative on $65 \%$ of the trials, and in the Incongruent treatment on $35 \%$ of the trials.

For each subject in the coacting condition there was a yoked control subject in the Alone condition. This subject heard the same paid participant as the coacting subject. The Alone subject, of course, did not have access to the actual choices of the coacting subject. Of the 19 subjects in each of the four cells, ten received the frequent light on the right and nine received it on the left.

\section{RESULTS}

Figure 3 shows the data for isolated and coacting subjects making decisions in the presence of congruent and incongruent cues. Again, we note an initial indifference between the alternatives, and a gradual reduction 


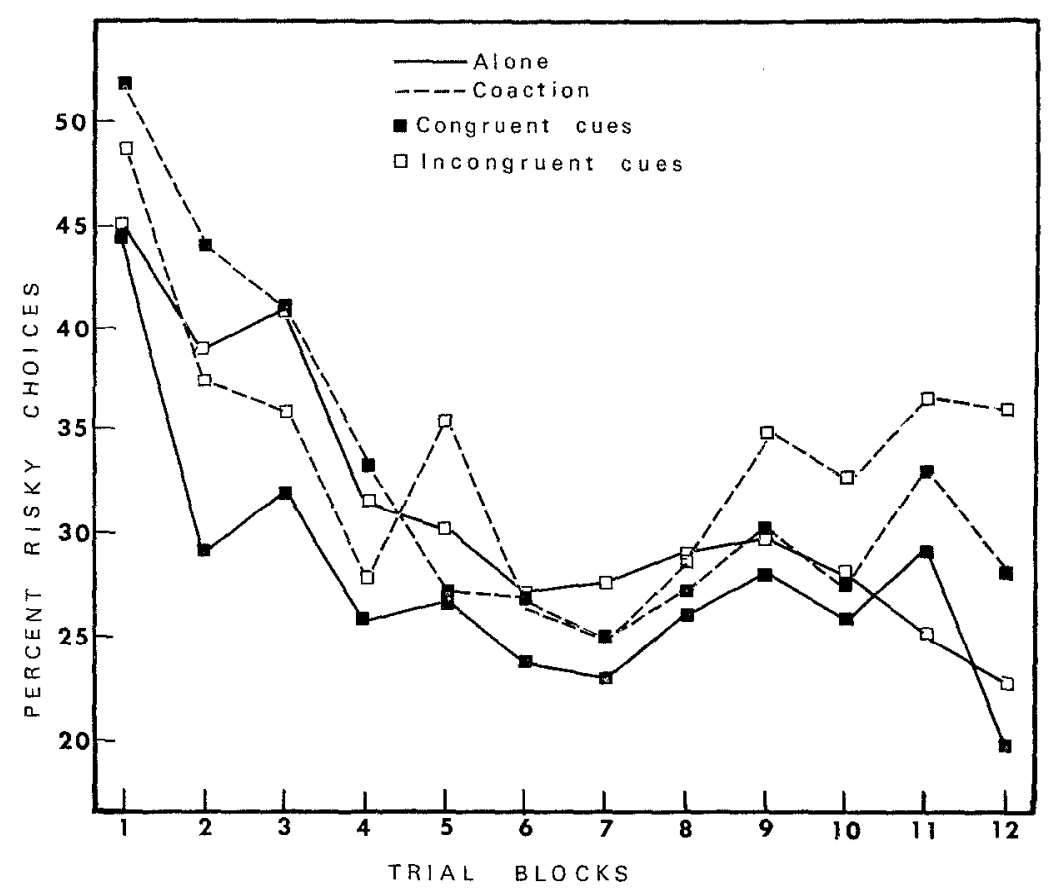

Fig. 3. The effect of coaction and congruent and incongruent cues on the percent of risky choices.

in choices of the risky alternative. The analysis of variance on these results shows a significant trial-block effect ( $F=24.84,11$ and $792 \mathrm{df}, p<$ $.001)$ with the first and second 180-trial blocks also significantly different from each other across all treatments and conditions $(F=90.45,1$ and $792 d f, p<.001)$. Also significant is the three-way interaction among the effects of coaction, cues, and halves of the experimental session ( $F=$ $21.67,1$ and $792 d f, p<.001$ ). Table 3 summarizes these results for the first and the second halves of the experiment. It is immediately apparent that the knowledge of others' choices does not produce simple effects. Let us first consider the effects of these cues when there are no coactors and the subject works in isolation. It seems that the individual's choice behavior is affected as we would expect, but primarily on the first 180 trials. That is, the knowledge of others' choices delayed the subject in reaching an asymptote. We note from Table 3 that on the second 180 trials isolated subjects working with congruent cues have nearly the same level of risk as those working with incongruent cues (25.1 vs. 26.8). The effect of cues in coaction is different. For subjects working in coacting groups it seems that the knowledge of others' choice affects not so 
TABLE 3

The Effects of Coaction and of Coactors' Choices on Decision

\begin{tabular}{lcc}
\hline \multicolumn{1}{c}{ Condition and treatment } & First 180 trials & Second 180 trials \\
\hline Alone: & 33.0 & 26.0 \\
Congruent cues & $30.4_{\mathrm{bcd}}{ }^{*}$ & $25.1_{\mathrm{d}}$ \\
Incongruent cues & $35.5_{\mathrm{ab}}$ & $26.8_{\mathrm{d}}$ \\
Coaction: & 36.4 & 29.8 \\
Congruent cues & $38.4_{\mathrm{a}}$ & $28.5_{\mathrm{bcd}}$ \\
Incongruent cues & $34.5_{\mathrm{abc}}$ & $31.1_{\mathrm{bed}}$ \\
Congruent cues (both treatments) & 34.4 & 26.0 \\
Incongruent cues (both treatments) & 35.0 & 29.8 \\
\hline
\end{tabular}

* Means having different subscripts are different at the .05 level by a Newman-Keuls comparison.

much the early levels of risk, but change over time. The curves for the coacting subjects intersect, while those for subjects working alone diverge initially and then converge. Looking at the differences between the first and second 180-trial sets we note that with congruent cues the coacting subjects drop from the first to the second half of the session by $9.9 \%$, while with inrongruent cues by $3.4 \%$. Thus, one might say that in the presence of coactors ihe subject's knowledge that their preferences disagree with his prevents him from reaching as low an asymptote as when their preferences agree with his. It is clear from Figure 3 that in coaction, subjects confronted with incongruent cucs tend to increase their risky choices beginning with trial-block 7 , showing that they are finally beginning to agree with their coactors. For subjects working in isolation, however, we noted that agreement with information provided by others takes effect rather earlier.

\section{DISCUSSION}

We have argued that there are probably many antecedent conditions which are sufficient for producing differences between individual and group decision-making that involve risk. One of the simplest of these antecedents must surely be the sheer physical presence of other individuals; for whatever processes are implicated in the group situation, at the very least, the joint presence of the group members is obviously a primary component. And with respect to the variable of presence of coactors and of spectators, it was shown that individual choice behavior under risk is sensitive to social facilitation effects. As predicted by the drive theory of social facilitation, when there is a dominant response, as there was here in the case of preference for the conservative alternative, the presence of 
others enhances its emission. The data from both experiments indicate that while individuals making decisions alone seem to prefer the conservative alternative, their preference becomes more pronounced in the presence of spectators or coactors.

But the direction of this effect together with data on group decisionmaking leads one to conclude that a simple social facilitation effect cannot account for the differences found between individual and group decisionmaking. Nor does it appear that information provided by others as to their choice is sufficient to produce shifts toward conservatism or toward risk in groups. The processes of imitation or conformity do not affect individual choice behavior in ways that would readily explain group shifts.

It is surprising, especially in the light of the value theories of risky shift (Brown, 1965; Nordhøy, 1962; Stoner, 1968), that knowledge about others' decisions had such a weak effect on the subjects' choices. According to Brown (1965), for instance, shifts toward risk occur by virtue of the following psychological process:

Perhaps riskiness is indeed an American value and each individual answering the Stoner problems means to be at least as risky as people like himself. . . . However, the individual who has not talked with anyone about the problems cannot know how to be truly risky because risk is relative to a group norm and the location of the norm on the scale of probabilities is not known. Each man, on his own, guesses the norm to be at or below his own selection. When individuals talk together and disclose their decisions the actual distribution is made known. Those who find themselves below the mean of the six members of the group discover that they are failing to realize the ideal of riskiness that they have thought they were realizing. Consequently they feel impelled to move in a risky direction both in accepting the decision of the group and in changing their private opinions. [p. 701]

We do have some information in Experiment I about the subjects' estimates of their own and others' levels of risk. Since we are primarily interested in those subjects who did not have access to others' choices, we shall first deal with these cstimatcs for the II, IA, and IC groups combined. The estimates of the subjects' own risk level were $38.1 \%$ in the .6-.4 condition and 23.2\% in the $.8-.2$ condition. The estimates of what "an average student at the University of Michigan" would do were $38.3 \%$ and $26.1 \%$, respectively. Neither of these differences approaches significance. The subjects' estimates of their own risk levels also do not differ from their estimates of "a professional gambler's" risk level. These latter averages were $39.5 \%$ for the $.6-.4$ condition and $26.3 \%$ for the $.8-.2$ condition. But there were also no systematic differences between subjects' estimates of their own and others' choices even when they had information about two other subjects. In the IG treatment, the .6-.4 subjects thought they were a bit riskier than the average student ( $46.8 \%$ for own 
risk and $44.0 \%$ for others' risk), and the $.8-2$ subjects thought they were more conservative than the average student ( $26.9 \%$ for own risk and $30.5 \%$ for others' risk). These differences did not approach significance.

The most interesting results in these estimates is that in every condition and every treatment there was a consistent underestimation of one's own actual level of risk. Thus, for instance, if we look only at the last 180 trials, the average actual percentage of risky choices in the II, IA, and IC treatments was $45.5 \%$ for the $.6-.4$ condition and $27.8 \%$ for the $.8-.2$ condition. ${ }^{*}$ In the first condition the subjects in the II, IA, and IC treatments underestimated their actual risk by $7.4 \%$ and in the second by $4.6 \%$. The first underestimation is significant at the .001 level $(t=3.00)$, and the second just fails to reach the acceptable level $(t=1.91)$.

Although the subjects' estimates of their own actual risk level are numerically inaccurate, they are accurate on a relative basis. The correlations between actual and estimated levels of own risk range from .66 to .90 . The accuracy of the subjects' relative risk level is most pronounced when they had access to others' decisions, as in the IG treatment. If, for each group in the IG treatment, we identify the member who was the highest, the member who was moderate, and the member who was the lowest in risk on trials when he worked independently, we can determine whether these latter three categories of subjects perceive the risk levels of their peers in the same manner. The results are quite clear here. Independently of whether the individual is high, medium, or low in initial risk, his judgment of peers' risk is the same. However, as we would expect from the correlation coefficients reported above, subjects high in relative risk attribute higher risk to themselves than members moderate or low in risk. This is true in the .6-.4 as well as in the .8-.2 condition. Moreover, the same comparison was made in the random "groups" composed of the II subjects, yielding the same results, although somewhat weaker. Hence, as we would expect, the between-subject variance of the subjects' estimates of their own level of risk is consistently larger than that of their estimates of others. This is, of course, more pronounced in the IG groups where there was an opportunity for a comparison. In the IG groups for both stimulus conditions combined the variance of subjects' estimates of their own risk was 381.6 while the variance of their estimates of others' risk was 210.6, a difference significant at the .05 level $(F=1.81,48$ and $48 d f)$. For the II, IA, and IC treatments these variances are 406.7 and 243.8 , respectively, also significantly different at the .05 level $(F=1.67$, 84 and $84 d f$ ).

${ }^{4}$ The subjects were instrucled to estimate the percentages of their choices over the entire session in the II, IA, and IC groups. Subjects in the IG treatment estimated their choices on the first 180 trials alone, when they worked independently. 
The fact that there are no differences between the estimates of subjects' own risk and the risk of their peers disagrees with the conjectures advanced by Brown (1965) and with data reported by Hinds (1962) and by Wallach and Wing (1968), who asked subjects to indicate the response to life dilemmas made by "the majority of your fellow students." Both Hinds and Wallach and Wing found that these subjects believed others to have lower risk levels. These comparisons, however, are between the actual level of risk of the given subject (as measured by his responses to life dilemmas) and his estimates of others' responses. In the present experiments, too, there was a similar difference. The subjects did in fact estimate others to be more conservative than they themselves actually were. But, of course, they estimated others to be no more risky and no more conservative than they estimated themselves to be.

It should be noted that subjects in the Wallach and Wing study knew that they would be asked about others' responses at the time they were giving their own, a condition inviting social comparison. The data in their study were collected by means of a mail questionnaire, and the respondents must have seen the entire instructions before giving answers. Whether the same estimates of others' risk would be obtained if the subjects were not required to give their own choices is a question which can only be answered by further research. Another question which must await further research and which is suggested by the results of our Experiment $I$ is whether we can assume the perception of the subject's own risk level to be identical to his actual risk-taking behavior. The value theory of risk and the research associated with these studies, of course, hold that the subject perceives his own risk-taking tendencies accurately. Our data contradict this assumption, at least for binary choices.

The data in Experiment II are in still greatcr disagrcement with the value theory of risk. This theory predicts that when the individual discovers that his risk level is lower than that of others, he should make the appropriate adjustment upward to meet the ideal. The conditions of this hypothesis are met for subjects who received incongruent cues. These subjects discovered that others chose the risky alternative on $65 \%$ of the trials. Yet the effect of this knowledge was numerically negligible. It would seem, therefore, that the value theory of risky shift does not apply to the present situation.

We are led to conclude that the significant antecedent conditions for risky or conservative shifts for tasks such as those in the above experiments are to be found in the process of interaction among the group members as they come to decisions. One of the critical features of this interaction must be the process by which a group reconciles the choice or risk preferences of the individual members. The existence of differ- 
ences in the members' preference for one alternative or the other is likely to generate discussion among them leading to the group choice. Implicit or explicit in this process is a rule for combining individual preferences into a single group choice. Majority rule, oligarchy, or taking turns, etc., are among the various rules which may be used in group decision-making. Research on risky shift has paid little attention thus far to the nature of these implicit or explicit rules of group decision-making under risk and uncertainty.

It is also evident from the above experiments, however, that whatever are the significant processes of group decision-making, they must be sufficiently robust to overcome the effects of social facilitation or imitation. In previous studies (Zajonc et al., 1968, 1969) we thought that group shifts could be explained without assuming changes in individual risk preferences or choice behavior. It is now clear that individual choice behavior is indeed affected by rather elementary social conditions, and it will become necessary for future research to determine the way in which the complex component processes of group decision-making interact with the simpler processes of social facilitation or imitation.

\section{REFERENCES}

Bateson, N. Familiarization, group discussion, and risk taking. Journal of Experimental Social Psychology, 1966, 2, 119-129.

Br.ANK, A. Effects of group and individual conditions on choìce behavior. Joumal of Personality and Social Psychology, 1968, 8, 294-298.

Brown, R. Social psychology. New York: Free Press, 1965.

Burns, J. F. An extremity-variance analysis of group decisions involving risk. Unpublished doctoral dissertation, Massachusetts Institute of Technology, 1967.

Cottrest, N. B., Rrtthe, R. H., \& WACK, D. L. The presence of an audience and list type (competitional or noncompctitional) as joint determinants of performance in paired-associated learning. Joumal of Personality, 1967, 35, 425434.

Flanders, J. P., \& Thistlethwarte, D. L. Effects of familiarization and group discussion upon risk taking. Journal of Personality and Social Psychology, 1967, 5; 91-97.

Goldmax, C. An examination of social facilitation. Unpublished manuscript, University of Michigan, 1967.

Hunds, W. C. Individual and group decisions in gambling situations. Unpublished master's thesis, School of Industrial Management, Massachusetts Institute of Technology, 1962.

Kogax, N., \& WALIACH, M. A. Bisk taking as a function of the situation, the person, and the group. In New directions in psychology III. New York: Holt, 1967. Pp. $111-278$.

Marovis, D. Individual and group decisions involving risk. Industrial Management Review, 1968, 9, 69-75.

Marquis, D. C., \& Rerrz, H. J. Uncertainty and risk taking in individual and group decisions. 1968. Submitted to Behavioral Science. 
NonDHøy, F. Group interaction in decision-making under risk. Unpublished master's thesis, Massachusetts Institute of Technology, School of Industrial Management, 1962.

Pruitx, D. G., \& Teger, A. I. The risky shift in group Letting. Technical Report No. 4, 1968, State University of New York at Buffalo.

Rabow, J., Fowler, F. J., Jr., Bradford, D. L., Hofeiler, M. A., \& Shrbuya, Y. The role of social norms and leadership in risk taking. Sociometry, 1966, 29, $16-27$.

Rettic, S., Johnson, F., \& TurofF, S. J. Group responsibility, affiliation, and ethical risk taking. Paper read at American Psychological Association convention, Washington, D.C., September, 1967.

StONER, J. A. F. Risky and cautious shifts in group decisions: The influence of widely held values. Journal of Experimental Social Psychology, 1968, 5, 442-459.

Strain, G. S. Social facilitation of oddity preferences in children. Paper presented at the Midwestern Psychological Association convention, Chicago, 1967.

TEger, A. I., \& Pruitr, D. G. Components of group risk taking. Joumal of Experimental Social Psychology, 1967, 3, 189-205.

Wallach, M. A., Kogan, N., \& BEM, D. J. Group influence on individual risk taking. Journal of Abnormal and Social Psychology, 1962, 65, 75-86.

Wallach, M. A., Kogan, N., \& Bem, D. J. Diffusion of responsibility and level of risk taking in groups. Journal of Abnormal and Social Psychology, 1964, 68, 263-274.

WALlach, M. A., \& WrNG, JR., C. W. Is risk a value? Journal of Personality and Social Psychology, 1968, 9(1), 101-106.

Zajonc, R. B. Social facilitation. Science, 1965, 149, 269-274.

Zajonc, R. B., \& SAles, S. M. Social facilitation of dominant and subordinate responses. Joumal of Experimental Social Psychology, 1966, 2, 160-168.

Zajonc, R. B., Wolosin, R. J., Wolosin, M. A., \& Sherman, S. J. Individual and group risk-taking in a two-choice situation. Journal of Experimental Social Psychology, 1968, 4, 89-106.

Zajonc, R. B., Wolosin, R. J., Wolosin, M. A., \& Sherman, S. J. Group risktaking in a two-choice situation: Replication, extension, and a model. Journal of Experimental Social Psychology, 1969, 5, 127-140.

(Received August 16, 1968) 